\title{
Anomalies chromosomiques et anomalies morphologiques des spermatozoïdes
}

\author{
Marie-Roberte GUICHAOUA, Georges MERCIER, Jacqueline SAIAS, \\ Vincent ACHARD, Odile LACROIX, Catherine GUILLEMAIN, Cendrine SIRAUDIN
}

Laboratoire de Biologie de la Reproduction, Hôpital de la Conception, Marseille

EA 1784, Biogénotoxicologie et mutagenèse environnementale

\section{INTRODUCTION}

Bien que la morphologie des spermatozoïdes soit l'un des paramètres qui suscite le plus de contradictions lorsque l'on veut en apprécier sa valeur prédictive in vivo et in vitro, il faut admettre que le pourcentage de spermatozoïdes anormaux et un certain nombre d'anomalies monomorphes des spermatozoïdes peuvent avoir une réelle valeur pronostique. II est clair que deux catégories de tératozoospermie apparaissent à l'analyse morphologique du sperme, les tératospermies polymorphes et les tératospermies monomorphes, qui posent des problèmes extrêmement différents de pronostic et de prise en charge. Avant de corréler les anomalies cytogénétiques des spermatozoïdes à la morphologie, il est indispensable de rappeler les difficultés que présente la réalisation d'un spermocytogramme qui se traduit par d'énormes écarts d'interprétation d'un laboratoire à l'autre, et d'insister sur la nécessité d'une standardisation de la préparation et de l'analyse des lames, du système de classification utilisé et du rendu des résultats.

En ce qui concerne l'analyse cytogénétique des spermatozoïdes, nous nous limiterons dans cet exposé aux anomalies de nombre des chromosomes dans les spermatozoïdes de patients dont le caryotype somatique est normal. Pour chaque patient, nous réalisons une triple $\mathrm{FISH}$ avec les sondes spécifiques des chromosomes $X$, $Y$ et un autosome (chromosome 9), afin de pouvoir distinguer les spermatozoïdes diploïdes des spermatozoïdes aneuploïdes et de pouvoir localiser les absences de cytodiérèse au cours de la première ou de la deuxième division méiotique. Nous complétons cette analyse avec une double FISH avec des sondes spécifiques de deux autosomes (chromosomes 8 et 18).

Au cours de cet exposé, nous parlerons très brièvement des anomalies chromosomiques dans les tératozoospermies polymorphes pour faire le point sur une anomalie morphologique fidèlement corrélée dans sa forme homogène à l'infertilité masculine, et surtout à la présence d'un génome quantitativement anormal alors que le caryotype somatique est normal. II s'agit du syndrome des spermatozoïdes macrocéphales (SM). Nous analyserons les problèmes que posent les formes partielles de ce syndrome.

\section{LES TÉRATOZOOSPERMIES POLYMORPHES}

Les tératozoospermies polymorphes ne sont pas de bons indicateurs d'anomalies chromosomiques des spermatozoïdes, les résultats de plusieurs études cytogénétiques montrent soit une absence significative de corrélation entre la fréquence des dysosmies et la tératozoospermie polymorphe [9], soit une augmentation modérée des anomalies chromosomiques dans ces spermes qui ne justifie pas une surveillance particulière de la grossesse [7]. II faut aussi retenir que les anomalies chromosomiques de nombre peuvent être présentes dans les têtes de spermatozoïdes de toutes les tailles et de toutes les formes, des spermatozoïdes diploïdes pouvant être d'apparence tout à fait normale [3]. Ainsi,

\section{Correspondance :}

Dr Marie-Roberte GUICHAOUA - Laboratoire de Biologie de la Reproduction, Hôpital de la Conception, 147 Boulevard Baille, 13387 Marseille - Email mguichaoua@ap-hm.fr 
l'augmentation significative des aneuploïdies et polyploïdies dans le sperme semble plutôt restreinte à une catégorie de tératozoospermie monomorphe se manifestant au niveau de la tête de spermatozoïdes.

\section{LE SYNDROME DES SPERMATOZOÏDES MACROCÉPHALES}

Si le syndrome des spermatozoïdes macrocéphales (SM) typique, tel qu'il a été décrit par Nistal en 1977, pose aujourd hui peu de problèmes quant à son origine génétique et son implication dans l'infertilité masculine, il n'en est pas de même des formes partielles de ce syndrome dont nous ne savons pas si elles sont la cause ou une association fortuite de troubles de la reproduction, et dont la fréquence est nettement supérieure à la forme classique initialement décrite. Il est donc évident qu'il existe non pas un, mais des syndromes SM. Nous allons dans cet exposé décrire le syndrome SM classique ainsi que les progrès qui ont été faits dans la connaissance de sa physiopathologie, nous en décrirons ensuite les étiologies, les différentes formes que nous avons observées, et leur prise en charge en Assistance Médicale à la Procréation (AMP).

\section{Les syndromes des spermatozoïdes macrocéphales de types 1, 2 et 3}

Ce syndrome est caractérisé par l'association de signes morphologiques très caractéristiques qui permettent d'en faire aisément le diagnostic : une augmentation du volume de la tête d'un facteur 4 qui résulte de la présence d'un noyau géant, une forme très irrégulière de la tête et de l'acrosome, et la présence inconstante de plusieurs flagelles (jusqu'à 4). L'Hybridation in situ sur les spermatozoïdes (FISH pour Fluorescent In Situ Hybridization) révèle une absence de cytodiérèse survenant principalement au cours de la méiose, mais il n'est pas exclu que ces événements puissent aussi se produire au cours des divisions mitotiques spermatogoniales. Toutes les études par FISH du complément chromosomique des spermatozoïdes de ces patients montrent la coexistence de spermatozoïdes tétraploïdes, diploïdes, et porteurs d'aneuploïdies complexes à des taux élevés. En ce qui concerne les spermatozoïdes diploïdes, la FISH révèle que l'absence de division cellulaire survient préférentiellement au cours de la première division méiotique, mais peut aussi se produire au cours de la deuxième division.

L'analyse automatisée de la surface des spermatozoïdes nous a permis de montrer que la surface moyenne des spermatozoïdes dans le syndrome SM était de $30,4 \mu \mathrm{m}^{2}$ $\pm 9,8 \mu \mathrm{m}^{2}$ (DS), alors que la surface moyenne des spermatozoïdes d'un sujet normal varie chez nos témoins de 11,9 à $13,5 \mu m^{2}\left( \pm 1,9\right.$ à $\left.2,8 \mu m^{2}\right)$ [1]. Cette analyse montre non seulement une augmentation importante de la surface moyenne des spermatozoïdes dans le syndrome SM mais aussi une plus grande dispersion des surfaces que chez les témoins, ce qui témoigne de la présence de toutes les tailles intermédiaire entre les tailles normales et celles des macrocéphales chez ces patients. Enfin, l'analyse détaillée de la morphologie des SM révèle une nette prédominance de SM de forme très irrégulière.

D'autres aspects biologiques de ce syndrome ont été clairement décrits par Escalier [6]. Dans le syndrome de type 2, la spermatogenèse s'arrête précocement au cours de la spermiogenèse, dans le syndrome de type 3 , les spermatozoïdes sont présents mais montrent une réduction de la croissance de l'axonème qui conduit à une désorganisation des structures périaxonémales. Tandis que le type 2 est caractérisé par l'absence de spermatozoïdes, une oligoasthénospermie sévère accompagne les types 1 et 3 . Les conséquences cliniques de ce syndrome se manifestent chez le patient dont les spermatozoïdes sont incapables de féconder en raison de l'asthénospermie et des anomalies de l'acrosome, et chez les embryons dont les anomalies chromosomiques sont responsables d'arrêts précoces du développement embryonnaire [8]. Un nouvel aspect du syndrome SM a été décrit par Benzacken et al. [2] dans lequel apparaissent des morts périnatales dans la fratrie du patient.

L'origine génétique de ce syndrome a été suspectée sur l'aspect homogène des lésions dans un même éjaculat, sur la présence fréquente d'une consanguinité parentale, et d'une histoire familiale d'infertilité (chez la moitié des patients). Etant donné le grand nombre de facteurs et d'enzymes normalement accumulés au cours du stade pachytène et nécessaires pour déclencher la première division de la méiose, de nombreux gènes semblent pouvoir être impliqués dans la survenue du syndrome SM [6]. Au cours de la division cellulaire, les mouvements des organites cellulaires nécessitent la déstabilisation du réseau de microtubules et la présence de kinases mitotiques qui interagissent avec les protéines associées à des organites phosphorylés. Récemment, Dieterich et al. [5] ont identifié une mutation du gène AURKC, c.144delC, qui conduit à la formation d'une protéine tronquée dépourvue du domaine kinase. Un grand nombre de gènes sont communs aux cycles cellulaires mitotique et méiotique, leurs mutations peuvent expliquer l'association du syndrome SM et des morts périnatales observées dans la fratrie des patients.

Les mécanismes impliqués dans la physiopathologie de ce syndrome qui associe une absence de cytokinèse et de cytodiérèse à une ségrégation aléatoire des chromosomes sont encore obscurs. On observe dans le spermatocyte au stade pachytène un défaut de répartition des organites cytoplasmiques tels que l'appareil de Golgi et des centrosomes, la cellule ne peut pas construire un fuseau bipolaire et n'évolue pas vers la métaphase, l'anaphase et la télophase sont absentes. Un grand nombre de ces spermatocytes sont arrêtés par les points de contrôle méiotiques, mais quelques cellules échappent à ces contrôles et donnent naissance à des spermatides géantes puis des spermatozoïdes macrocéphales. L'aneuploïdie 
associée à la polyploïdie du syndrome des SM résulterait d'une partition inégale des chromosomes ou des chromatides au cours de la méiose, les fuseaux de division n'étant que partiellement fonctionnels tandis que la cellule est capable de réaliser l'une ou les deux divisions méiotiques. Il est possible aussi que la fixation des chromosomes aux fibres fusoriales soit défectueuse en raison de l'existence de jeux incomplets de kinétochores.

Ainsi, l'oligospermie constamment observée chez ces patients résulterait probablement à la fois d'une réduction de l'activité mitotique spermatogoniale et d'un blocage méiotique des spermatocytes polyploïdes ou porteurs d'aneuploïdies complexes. Ces formes typiques sont rares et ne posent pas de problèmes diagnostiques. Quant à leur prise en charge, si la majorité des équipes excluent ces spermes d'une AMP, Kahraman et al. [8] ont réalisé des ICSI avec des SM. Les résultats obtenus, taux bas de fécondation et de grossesses, ne sont pas surprenants. La même équipe a ensuite réalisé un diagnostic préimplantatoire (DPI) sur les embryons obtenus avec de tels spermatozoïdes : $46,4 \%(32 / 69)$ des embryons obtenus étaient porteurs d'anomalies chromosomiques, plus du tiers de ces anomalies étant des trisomies, les autres, des aneuploïdies complexes. Le DPI a permis d'obtenir un taux de grossesse de $33 \%(7 / 21)$ et un taux d'implantation de $25 \%$. Une seule de ces grossesses s'est interrompue spontanément à 8 semaines, mais le caryotype du fœtus était normal $(46, X X)$.

\section{Les formes partielles du Syndrome des spermatozoïdes macrocéphales}

Beaucoup plus intéressantes sont les formes partielles du syndrome SM chez les hommes consultant pour infertilité $[1,10]$. Ces formes sont caractérisées par la coexistence lors de l'analyse du sperme de SM et de spermatozoïdes de taille normale. Cependant, l'analyse automatisée de la surface des spermatozoïdes met en évidence des spermatozoïdes de toutes les tailles entre le spermatozoïde de taille normale et le SM ; elle montre par ailleurs une distribution intermédiaire entre celle des témoins et celle des patients porteurs du syndrome SM de type1et 3. La surface moyenne des spermatozoïdes est parfois normale, elle varie chez nos patients de 11,8 à $18,7 \mu \mathrm{m}^{2}$ mais les DS sont toujours augmentées, variant de 4,2 à $6,3 \mu \mathrm{m}^{2}$, et les histogrammes mettent en évidence soit un étalement des surfaces vers les grandes valeurs, soit la présence d'une sous-population correspondant au clone macrocéphale. La FISH sur les spermatozoïdes montre la présence de spermatozoïdes diploïdes et aneuploïdes et de rares spermatozoïdes tétraploïdes, mais aussi la présence de spermatozoïdes normaux avec les sondes utilisées. Nous avons remarqué que le pourcentage de spermatozoïdes porteurs d'anomalies chromosomiques était toujours supérieur au pourcentage de spermatozoïdes macrocéphales, ceci se comprend aisément lorsque l'on regarde les courbes de surfaces des spermatozoïdes.
Certains spermatozoïdes de taille sub-normale sont classés parmi les spermatozoïdes normaux, nous avons aussi signalé plus haut que des spermatozoïdes diploïdes pouvaient avoir une taille normale [3]. Nous avons également décrit, associées à une forme partielle du syndrome SM, des morts périnatales dans la fratrie d'un patient [1].

D'autres caractéristiques distinguent les formes partielles des formes totales. Tout d'abord, nous avons montré que, contrairement aux formes homogènes, il pouvait $y$ avoir dans ces formes partielles une prédominance de SM de forme régulière et ne contenant qu'un seul flagelle [10]. Puis, la FISH sur les spermatozoïdes nous a permis de mettre en évidence des formes dans lesquelles prédomine une absence de cytodiérèse au cours de la deuxième division méiotique, ce qui n'a pour l'instant jamais été décrit dans les formes totales [1].

Il est actuellement impossible de donner la fréquence des formes partielles du syndrome SM. Nous n'avons pas encore défini le seuil de SM à partir duquel ce syndrome retentit sur la fonction de reproduction, le pourcentage de SM dans la population d'hommes infertiles consultant dans notre centre est de $1,26 \%$, calculé sur 7292 spermogrammes. L'évaluation de ce seuil est difficile, car le pourcentage de SM fluctue en fonction du cycle de l'épithélium séminifère, et qu'il semble augmenter progressivement avec le temps [4] probablement en rapport avec une altération progressive de la spermatogenèse chez ces patients. Nous n'avons pas encore assez de recul et de cas pour établir un lien entre ces formes partielles et les troubles de la reproduction : infertilité, diminution des taux de fécondation et de grossesse en FIV, fausses couches spontanées précoces, morts périnatales. Aucune naissance d'enfant porteur d'une anomalie chromosomique viable n'a encore été signalée.

Nous avons jusqu'à présent montré que des hommes infertiles, dont le taux de MS était égal ou supérieur à $20 \%$ dans au moins un spermogramme, avaient un pourcentage élevé d'anomalies chromosomiques [1]. Notre attention se porte maintenant vers les hommes dont le taux de SM se situe entre 10 et $20 \%$.

Une autre inconnue concerne l'origine de ce syndrome SM partiel, qui peut être d'origine génétique ou environnementale. Dans le cas d'une cause génétique, comment expliquer la coexistence. de SM et de spermatozoïdes de taille normale ? Il pourrait s'agir d'une mosaïque germinale résultant d'une mutation spontanée qui se produit dans une cellule germinale qui continue à se diviser [11] ou d'une mutation génique présente dans toutes les cellules mais qui s'exprimerait seulement dans un certain pourcentage de cellules germinales, en fonction des conditions testiculaires. Ces formes partielles peuvent aussi résulter de l'action de toxique environnementaux : fumée de cigarettes, chimiothérapie, pesticides. 
Quel est le risque pour l'embryon et quelle est la conduite à tenir vis à vis de ces couples qui bénéficient d'une AMP ? Nous savons que des spermatozoïdes, bien que de taille normale, peuvent être porteurs d'anomalies chromosomiques et être utilisés en ICSI, conduisant à la formation d'embryons porteurs d'anomalies chromosomiques. Le risque majeur pour ce couple étant l'interruption spontanée de la grossesse. La survenue de morts périnatales à répétition au sein de ces couples, ou la naissance d'enfants porteurs de trisomies viables, n'a encore jamais été signalée. Le déséquilibre chromosomique de l'embryon peut aussi être la conséquence directe de la mutation responsable du syndrome. Ainsi, un embryon dont le contenu génétique est initialement normal peut subir des non-disjonctions chromosomiques lors des premières divisions de segmentation. Nous n'avons pas suffisamment de recul pour définir une conduite à tenir pour ces couples. Si nous avons pris la décision dans notre centre d'exclure de toute tentative d'AMP les spermes montrant la présence d'un syndrome homogène, les formes partielles sont prises en charge, les couples bénéficiant d'un conseil génétique et d'une FISH sur les spermatozolldes. En cas de grossesse, nous préconisons une surveillance étroite, échographique et triple test.

\section{CONCLUSION}

Nous insistons sur l'hétérogénéité clinico-biologique du syndrome SM qui se manifeste au niveau des troubles de la reproduction présentés par les patients, de la morphologie des spermatozoïdes et dans leur composition cytogénétique. Alors que pendant des années, nous nous sommes limités à demander à juste titre un caryotype chez les hommes dont la numération des spermatozoïdes était inférieure à 10 millions par $\mathrm{ml}$, nous pensons qu'il est important maintenant de savoir prescrire une analyse chromosomique quantitative des spermatozoïdes par FISH. C'est un examen long et coûteux, dont les indications doivent être posées avec modération, et qui pourrait être complété ou parfois remplacé par l'analyse automatisée de la surface des spermatozoïdes.

\section{REFERENCES}

1. ACHARD V., PAULMYER-LACROIX O., MERCIER G. et al.: Reproductive failure in patients with various percentages of macronuclear spermatozoa : high level of aneuploid and polyploid spermatozoa. J. Androl., 2007 (sous presse).

2. BENZACKEN B., GAVELLE F.M., MARTIN-PONT B. et al. : Familial sperm polyploidy induced by genetic spermatogenesis failure : case report. Hum. Reprod., 2001, 16 : 2646-2651.

3. CELIK-OZENCI C., JAKAB A., KOVACS T. et al. : Sperm selection for ICSI : shape properties do not predict the absence or presence of numerical chromosomal aberrations. Hum. Reprod., 2004, 19 : 2052-2059.
4. DEVILLARD F., METZLER-GUILLEMAIN C., PELLETIER R. et al. : Polyploidy in large-headed sperm : FISH study of three cases. Hum. Reprod., 2002, 17: 1292-1298.

5. DIETERICH K., SOTO RIFO R., FAURE A.K. et al. : Homozygous mutation of AURKC yields large-headed polyploid spermatozoa and causes male infertility. Nat. Genet., 2007, 39 : 661-665. Epub 2007 Apr 15.

6. ESCALIER D. : Genetic approach to male meiotic division deficiency : the human macronuclear spermatozoa. Mol. Hum. Reprod., 2002, $8: 1-7$.

7. FAURE A.K., AKNIN-SEIFER I., FREROT G. et al. : Predictive factors for an increased risk of sperm aneuploidies in oligoastheno-teratozoospermic males. Int. J. Androl., 2007, 30:153162.

8. KAHRAMAN S., SERTYEL S., FINDIKLI N. et al. : Effect of $P G D$ on implantation and ongoing pregnancy rates in cases with predominantly macrocephalic spermatozoa. Reprod. Biomed. Online, 2004, 9 : 79-85.

9. RIVES N., SAINT CLAIR A., MAZURIER S. et al. : Relationship between clinical phenotype, semen parameters and aneuploidy frequency in sperm nuclei of 50 infertile males. Hum. Genet., 1999, $105:$ 266-272.

10. YUROV Y.B., SAIAS M.J., VORSANOVA S.G. et al. : Rapid chromosomal analysis of germ-line cells by FISH : an investigation of an infertile male with large-headed spermatozoa. Mol. Hum. Reprod., 1996, 2 : 665-668.

11. ZLOTOGORA J. : Germ line mosaicism. Hum. Genet., 1998, $102: 381-386$.

Manuscrit reçu : septembre 2007 ; accepté septembre 2007.

Communication au XXVIème Congrès de la SALF, décembre 2007, Colmar 